\title{
Dietary Patterns and Their Nutrients in Older New Zealand Adults ${ }^{+}$
}

\author{
Karen Mumme ${ }^{1}$, Cathryn Conlon ${ }^{1}$, Pamela von Hurst ${ }^{1}$, Mary Beatrix Jones ${ }^{2}$, Crystal Haskell- \\ Ramsay ${ }^{3}$, Welma Stonehouse ${ }^{4}$, Anne-Louise Heath ${ }^{5}$, Jane Coad ${ }^{6}$, Jamie de Seymour ${ }^{1}$ and \\ Kathryn Beck ${ }^{1, *}$ \\ 1 College of Health, Massey University, Auckland 0745, New Zealand \\ Department of Statistics, University of Auckland, Auckland 1010, New Zealand \\ 3 Department of Psychology, Northumbria University, Newcastle NE1 8ST, UK \\ 4 Health and Biosecurity Business Unit, Commonwealth Scientific Industrial Research Organisation \\ (CSIRO), Adelaide SA 5000, Australia \\ 5 Department of Human Nutrition, University of Otago, Dunedin 9016, New Zealand \\ 6 College of Science, Massey University, Palmerston North 4442, New Zealand \\ * Correspondence: k.1.beck@massey.ac.nz; Tel.: +64-9-414-0800 (ext. 43662) \\ + Presented at the 2019 Annual Meeting of the Nutrition Society of New Zealand, Napier, New Zealand, \\ 28-29 November 2019.
}

Published: 17 December 2019

Dietary patterns analyse how combinations of foods are eaten. Little is known about the dietary patterns of older adults living in New Zealand. This study explores nutrient differences within dietary patterns of older, community-dwelling adults (65-74 years) in Auckland.

Dietary data (109-item food frequency questionnaire) collected in the Research Eating, Activity and Cognitive Health (REACH) study ( $n=367,36 \%$ male) was collapsed into 57 food groups. Using principal component analysis followed by varimax rotation, two dietary patterns (DP) were extracted which explained $14 \%$ of the variance in dietary intake. Food group nutrients were obtained from the New Zealand FOODfiles ${ }^{\mathrm{TM}} 2016$ database. Trends in DP score and nutrients (standardised by energy intake) were investigated by linear regression analysis with consideration of sex.

DP1 is a 'healthy' pattern characterised by 'vegetables', 'nuts and seeds', 'legumes', 'meat alternatives', 'fruit', and 'fish and seafood'. Positive associations were noted with fibre, mono- and poly-unsaturated fats, vitamin A, beta-carotene equivalents, thiamine, folate, vitamin $\mathrm{C}$, vitamin $\mathrm{E}$, iron, potassium, magnesium $(p<0.001)$ were noted. Negative associations were observed with saturated fat $(p<0.001)$, riboflavin and calcium $(p=0.003)$.

DP2 is a 'western' style pattern characterised by 'processed meats', 'sauces and dressings', 'biscuits and cakes', 'takeaway food', 'confectionary', 'chocolate', 'vegetable oils', and 'cheese'. Positive associations were noted with carbohydrate and sodium $(p<0.001)$. Intakes of fibre, polyunsaturated fat, beta-carotene equivalents (females only), vitamin B6, folate, vitamin C, vitamin E, potassium, magnesium $(p<0.001)$ and mono-unsaturated fat $(p=0.003)$ were reduced with higher adherence to DP2.

DPs are supported by intakes of nutrients. This underlying nutrient knowledge can help explain mechanisms behind health outcomes. These DPs add evidence to dietary habits of the older New Zealand population and will be used further to examine associations with cognitive function and metabolic syndrome.

(C) 2019 by the authors. Licensee MDPI, Basel, Switzerland. This article is an open access article distributed under the terms and conditions of the Creative Commons Attribution (CC BY) license (http://creativecommons.org/licenses/by/4.0/). 